Review Article

\title{
Biological Significance of Urolithins, the Gut Microbial Ellagic Acid-Derived Metabolites: The Evidence So Far
}

\author{
Juan Carlos Espín, Mar Larrosa, María Teresa García-Conesa, \\ and Francisco Tomás-Barberán \\ Research Group on Quality, Safety and Bioactivity of Plant Foods, Department of Food Science and Technology, \\ CEBAS-CSIC, Campus de Espinardo, P.O. Box 164, 30100 Murcia, Spain
}

Correspondence should be addressed to Francisco Tomás-Barberán; fatomas@cebas.csic.es

Received 4 January 2013; Revised 15 April 2013; Accepted 17 April 2013

Academic Editor: David Heber

Copyright (C) 2013 Juan Carlos Espín et al. This is an open access article distributed under the Creative Commons Attribution License, which permits unrestricted use, distribution, and reproduction in any medium, provided the original work is properly cited.

\begin{abstract}
The health benefits attributed to pomegranate have been associated with its high content in polyphenols, particularly ellagitannins. This is also the case for other ellagitannin-containing fruits and nuts including strawberry, raspberry, blackberry, walnuts, and muscadine grapes. The bioavailability of ellagitannins and ellagic acid is however very low. These molecules suffer extensive metabolism by the gut microbiota to produce urolithins that are much better absorbed. Urolithins circulate in plasma as glucuronide and sulfate conjugates at concentrations in the range of $0.2-20 \mu \mathrm{M}$. It is therefore conceivable that the health effects of ellagitannin-containing products can be associated with these gut-produced urolithins, and thus the evaluation of the biological effects of these metabolites is essential. Recent research, mostly based on in vitro testing, has shown preliminary evidence of the anti-inflammatory, anticarcinogenic, antiglycative, antioxidant, and antimicrobial effects of urolithins, supporting their potential contribution to the health effects attributed to pomegranate and ellagitannin-rich foods. The number of in vivo studies is still limited, but they show preventive effects of urolithins on gut and systemic inflammation that encourage further research. Both in vivo and mechanistic studies are necessary to clarify the health effects of these metabolites. Attention should be paid when designing these mechanistic studies in order to use the physiologically relevant metabolites (urolithins in gut models and their conjugated derivatives in systemic models) at concentrations that can be reached in vivo.
\end{abstract}

\section{Introduction}

The health effects of pomegranate and pomegranate juice have been associated withthe high content in antioxidant polyphenols [1] and particularly in ellagitannins (punicalagins). This is also the case for many other ellagitannincontaining fruits and nuts such as strawberry, raspberry, blackberry, walnuts, and muscadine grapes $[2,3]$.

Both ellagic acid and ellagitannins have shown relevant biological effects in animal models and human studies which suggests potential preventive effects against chronic diseases such as cancer, diabetes, cardiovascular diseases, and neurodegenerative diseases [4]. These effects are associated with a multitarget action that involves antioxidant, antiinflammatory, and anticarcinogenic effects $[2,5]$. It is well established that ellagitannins and ellagic acid absorption is very low and that the unabsorbed compounds are further metabolized to urolithins by the gut microbiota in the colon [6-10]. This poor bioavailability and the extensive gut catabolism suggest that urolithins rather than ellagitannins or ellagic acid may be the actual bioactive molecules $[7,11]$.

Urolithins are dibenzopyran-6-one derivatives with different hydroxyl substitutions. Chemically they can be considered a combination of coumarin and isocoumarin (benzocoumarins) (Figure 1). They are produced from ellagic acid by the gut microbiota through the loss of one of the two lactones 

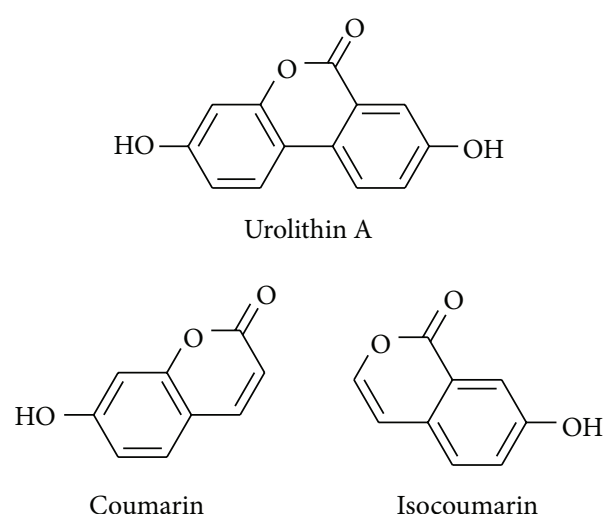

FIgURE 1: Chemical structure of urolithin A, a benzocoumarin.

present in ellagic acid (lactonase/decarboxylase activity) and by successive removals of hydroxyls (dehydroxylase activities) [12] (Figure 2).

Urolithins are not common molecules in nature, but they have been reported in plants rich in ellagitannins, as it is the case of Tamarix nilotica flowers [13] and Punica granatum leaves that contain urolithin M-5 (3,4,8,9,10-pentahydroxydibenzo[b,d]pyran-6-one) [14]. They have also been found in some herbivore-derived products, such as the castoreum produced by beavers [15], a product of interest for the perfume industry, and the Pteropi faeces (faeces of the squirrel Trogopterus xanthipes) that are used in traditional Chinese medicine [16]. Urolithins are also relevant constituents of shilajit, a soil-derived medicinal product used in Ayurvedic medicine [17].

The name urolithin was first given to two metabolites isolated from the renal calculus of sheep (Trifolium subterraneum has been reported as the cause of clover stone and might be a relevant source of ellagitannins) that were named urolithin A and urolithin B [18]. These two molecules coincided with pigments I and II previously described from castoreum and beaver glands [15].

The intake of large amounts of ellagitannins, and particularly of punicalagin, from Terminalia oblongata by cattle was associated with hepatotoxicity and nephrotoxicity [19]. However, the subchronic oral administration of large amounts of pomegranate ellagitannins was not toxic to rats where these compounds were found to be extensively metabolized to urolithins [20].

Since urolithins are ellagitannin-derived catabolites that can be absorbed and reach different tissues in the body, they have been suggested as the molecules potentially responsible for the biological effects observed as a consequence of the consumption of pomegranate or other ellagitannincontaining foods $[3,7,9,11]$. Here we review the state of the art in urolithin metabolites production by gut microbiota, their absorption, tissue distribution and pharmacokinetics, the cell and molecular mechanisms for their biological effects reported so far using different in vitro models, and the in vivo evidence in animals and humans.

\section{Urolithins Chemical Nature, Detection, and Identification}

Urolithins constitute a whole metabolic family produced by the opening and decarboxylation of one of the lactone rings of ellagic acid and the sequential removal of hydroxyls from different positions (Figure 2). After decarboxylation the first metabolite is urolithin M-5 (pentahydroxy-urolithin), and from this, several tetrahydroxy-urolithin isomers are produced by removal of one hydroxyl group from different positions (urolithin D, urolithin M-6). Trihydroxy-urolithins (urolithin C, urolithin M-7) were then produced after the removal of a second hydroxyl and dihydrox- urolithins (urolithin A and isourolithin A) after the removal of a third one. Monohydroxy-urolithin (urolithin B) was also detected, particularly in those cases in which isourolithin A was produced (Figure 2). Further degradation of urolithins to remove the second lactone ring has not been reported so far, although it should not be discarded. Urolithins show characteristic UV spectra that can be used for the identification of different hydroxyl-substitution patterns on the urolithin nucleus, as well as the further conjugation with methyl, glucuronic acid, or sulfate [21]. The UV spectra of urolithins in methanol exhibit two major absorption bands in the region of 240-400 $\mathrm{nm}$ (Figure 4). These are referred to as band I (300-380 nm) and band II (240-280 nm). In many cases, an additional band III (between 280 and $300 \mathrm{~nm}$ ) is also observed. Unfortunately, bands I and II cannot be associated with a specific ring of the urolithin nucleus [21]. Two groups of urolithin UV spectra were distinguished after a study of the plots of the different spectra: urolithins with a hydroxyl in the 9-position and those without hydroxylation in the 9-position (Figure 4). The occurrence of the hydroxyl at position 9, as is the case of isourolithin A, produces a hypsochromic shift in band I and an increase in the absorption of band II, displaying a maximum around $256 \mathrm{~nm}$, which is the main absorption band of the spectrum of urolithins with a hydroxyl in the 9position. Conjugation with glucuronic acid or sulfate also has measurable effects on the UV spectrum, and could be used as a diagnostic method for urolithin metabolites identification.

\section{Bioavailability, Metabolism, and Tissue Distribution of Pomegranate Ellagitannins and Their Metabolites}

Due to the potential health effects and the expectation raised by the high antioxidant activity of pomegranate juices that was thought to be due to punicalagins, punicalins and other ellagitannins [1, 22], a sensible step in the research was the determination of the absorption and metabolism of these compounds in animals and humans. The first study testing the bioavailability of pomegranate ellagitannins was carried out on rats, and this showed that after the intake of large amounts of pomegranate husk ellagitannins, the main metabolites detected in plasma and urine were urolithins $\mathrm{A}, \mathrm{B}$, and C (Figure 2) and smaller amounts of ellagic acid-dimethyl ether glucuronide [6]. Interestingly, a small amount of punicalagin was also detected in plasma and urine, 


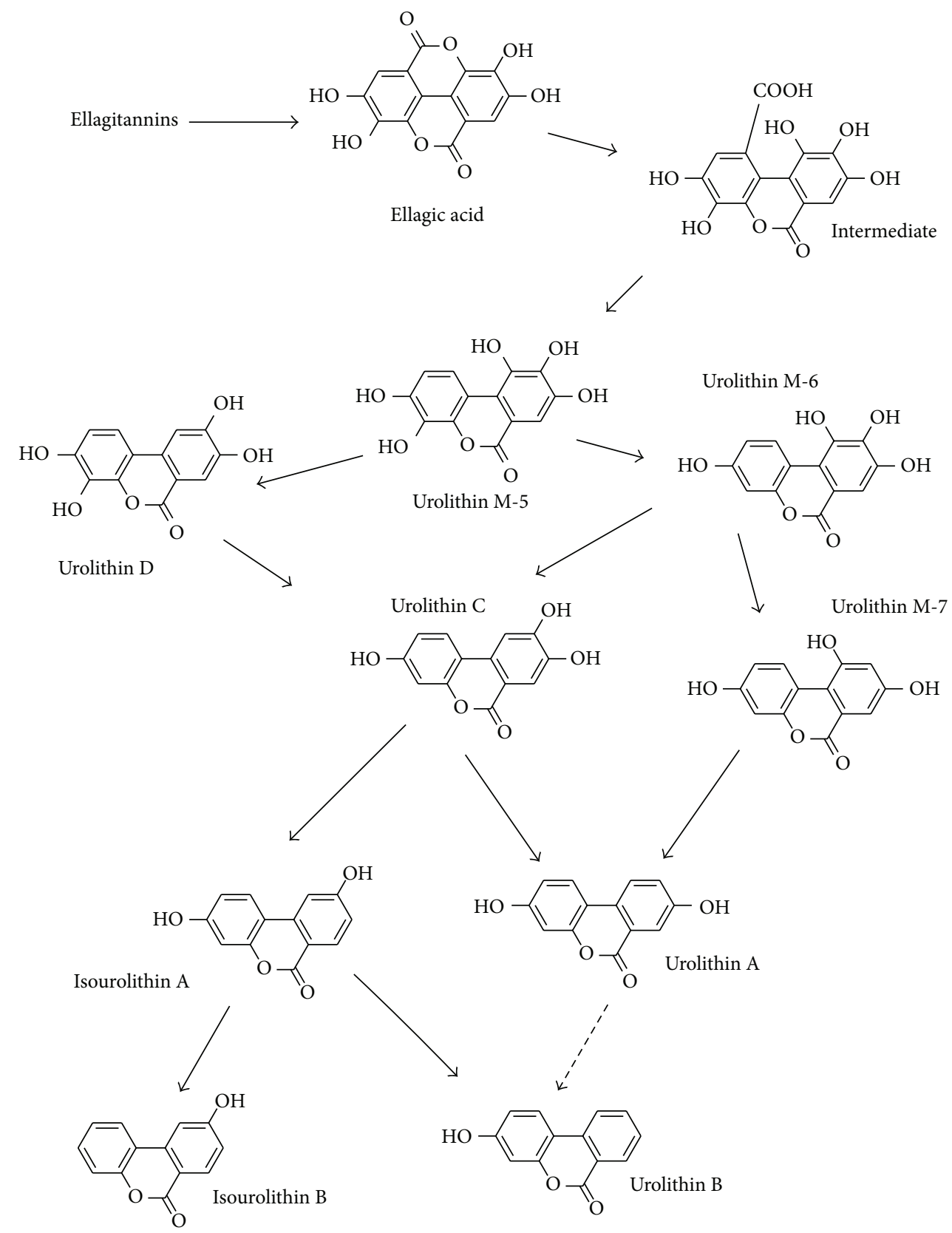

FIGURE 2: Gut microbiota metabolism of ellagitannins and ellagic acid.

particularly after a long period of intake, but this has not been confirmed in further studies in humans in which dietary relevant amounts of pomegranate ellagitannins were supplied [7].

Urolithin A was produced from ellagic acid, punicalagin, and an ellagitannin-rich walnut extract by fecal microbiota from six volunteers, demonstrating for the first time the production of urolithins by human gut microbiota [12]. In addition, a large interindividual variability was observed in this ex vivo experiment [12] in agreement with the results observed in vivo [7] suggesting that differences in the microbiota composition affect urolithin production and therefore the potential health effects after consumption of ellagitanninrich foods [12]. No correlation between urolithin production from ellagitannins and equol production from isoflavones was observed [12], and this indicated that different bacterial strains are involved in the gut metabolism of isoflavones and ellagitannins.

The Iberian pig was used as a model to study urolithin production from ellagitannins. This particular pig feeds on oak acorns that are very rich in ellagitannins, and this model was used to evaluate ellagitannin metabolism and tissue distribution [23] and to help in understanding the ellagitannin metabolism in humans. This study showed that 


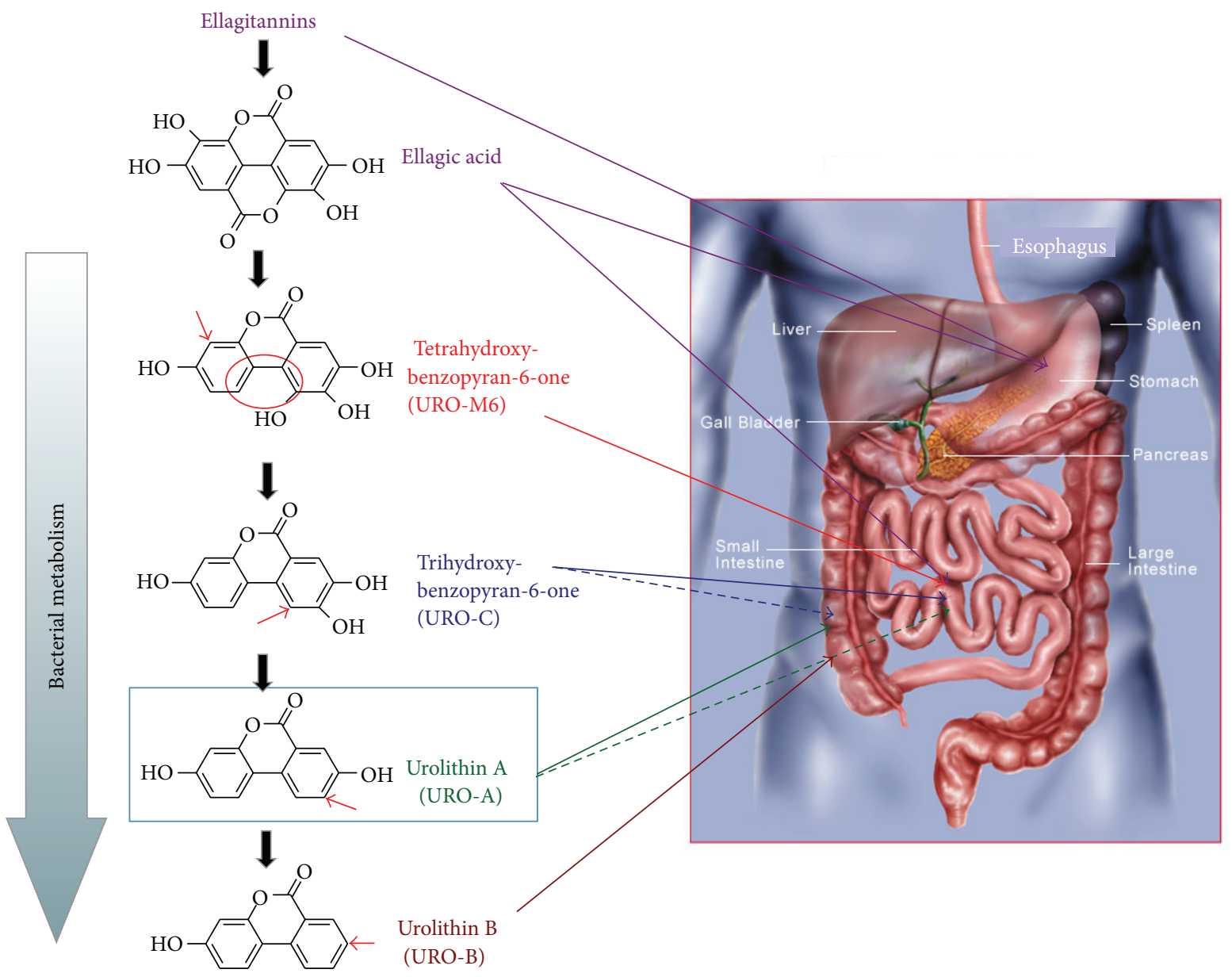

FIGURE 3: Sequential production of urolithins in the gut.

different urolithins were produced in the gut starting with tetrahydroxy-urolithin through the removal of one of the lactone rings of ellagic acid, and by sequential removal of hydroxyls to end with urolithins A and B (Figure 3). The analysis of plasma and urine samples showed that urolithin A glucuronide and sulfate were the main metabolites, with urolithin $\mathrm{C}$ and $\mathrm{B}$ glucuronides and sulfates as minor metabolites. Ellagic acid dimethyl ether glucuronide was also a significant metabolite. The analysis of the gall bladder and bile showed that tetrahydroxy-urolithin was absorbed in the first portion of the gut, and was detected in the liver, where it was conjugated and excreted with the bile to the small intestine. An entero-hepatic recirculation was clearly revealed in this animal model study [23]. Regarding the tissue distribution, urolithin metabolites only accumulated in the urine bladder and the gall bladder where they reached high concentrations, but they did not accumulate in any of the analyzed tissues (muscle, adipose tissue, kidney, liver, heart, etc.) [23].

The occurrence of low concentrations or urolithin metabolites in mouse prostate gland after pomegranate ellagitannins intake [24] and in human prostate after the intake of pomegranate juice and walnuts [25] has been reported. This last study is the only one available on the evaluation of the occurrence of ellagitannin metabolites in human tissues (prostate biopsies), and the occurrence of urolithin A glucuronide (2 $\mathrm{ng} / \mathrm{g}$ tissue) and traces of urolithin B glucuronide and ellagic acid dimethyl ether was reported [25].

There are no studies on the pharmacokinetics and tissue distribution of urolithins after their direct intake in humans. There is, however, indirect evidence of both aspects after the intake of ellagitannins or ellagic acid-rich food products. In the case of pomegranates or derived beverages, it is well established that ellagitannins are not absorbed when dietary doses are ingested and that the released ellagic acid is absorbed in the first part of the gastrointestinal tract and is detected as such in plasma with $C_{\max }$ concentrations around $100 \mathrm{nM}$ with $T_{\max } 1$ hour after the intake $[10,26]$. Ellagic acid conjugates have also been detected, and these include methyl ether and glucuronyl and sulphate conjugates. The most common metabolite found in urine and plasma is ellagic acid dimethyl ether glucuronide, which involves the methylation by COMT and then the glucuronidation by glucuronyl-transferase [3,7]. The peak plasma levels of urolithin A were $14-25 \mu \mathrm{M}$ depending on the volunteers. These metabolites started to appear in plasma 6-8 hours after the intake, which confirms urolithin production in the 
<smiles>O=c1oc2cc(O)ccc2c2ccc(O)cc12</smiles>

Urolithin A

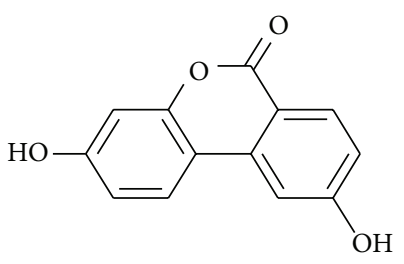

Isourolithin A

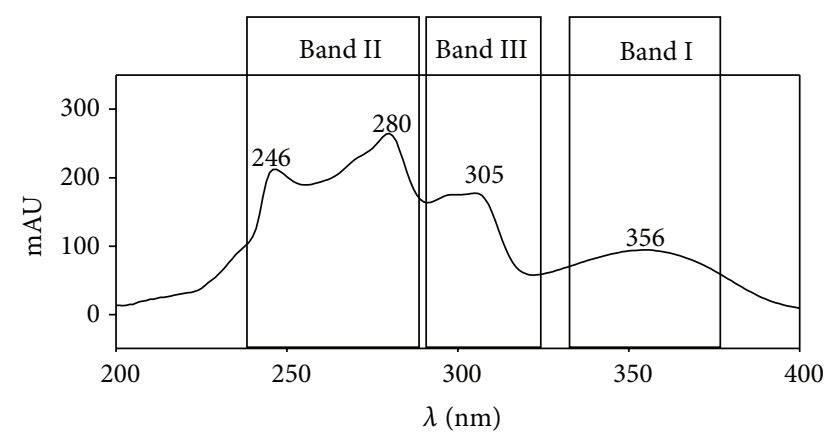

(a)

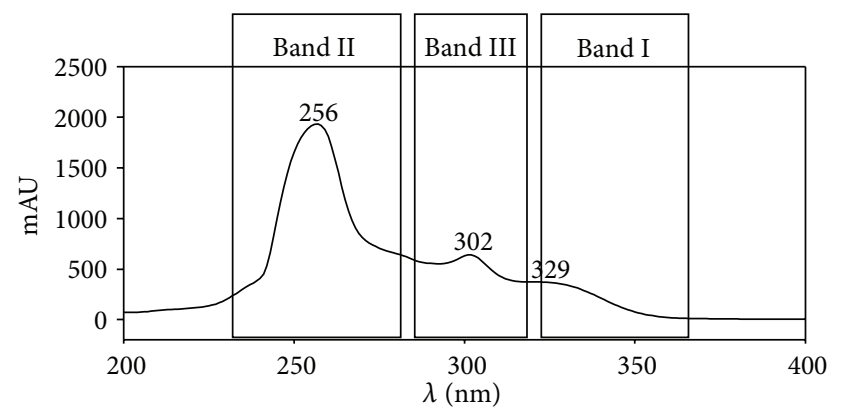

(b)

FIGURE 4: UV characteristic spectra of urolithins.

colon [7], and persisted in urine and plasma 48-72 hours after the ellagitannins intake showing an active enterohepatic recirculation $[3,7,9,12]$.

Seeram et al. [24] described the pharmacokinetics and tissue distribution of urolithins in mice after oral or intraperitoneal administration of chemically synthesized urolithin A (human equivalent dose, HED $\sim 85 \mathrm{mg}$ for a $70 \mathrm{~kg}$ person). Urolithin A peaked in plasma after $2 \mathrm{~h}$ and reached the highest concentrations in the prostate followed by the small intestine and colon, with a peak at $4 \mathrm{~h}$ [24]. Urolithin A, urolithin A sulfate, and methyl-urolithin A were mainly detected in the prostate gland whereas urolithin A glucuronide was primarily detected in liver and kidney tissues. Other studies with animal models, including rats and pigs, confirmed the urolithin production in the colon, and the sequential loss of hydroxyls when the metabolites advance in the intestinal tract $[3,12]$.

González-Sarrías et al. [25] reported the occurrence of urolithin metabolites and ellagic acid dimethyl ether glucuronide in prostate biopsies after the intake of pomegranate juice. This is the only study carried out so far to evaluate the occurrence of urolithin metabolites in human tissues. The concentration detected in the prostate gland was however low, although higher concentrations should not be discarded, as suggested by a parallel study carried out in rats [25] and also in agreement with the results obtained by Seeram et al. [24].

\section{Production of Urolithins by Different Animals}

The production of urolithins from ellagitannins has been reported in different animals (Table 1). Those animals that feed on bark and wood, as is the case of beavers and squirrels, produce urolithins in their gut, and they are present in their feces and in excretions/secretions as is the case of castoreum.

In addition, ruminants that feed on ellagitannin-rich fodders (oak leaves, Trifolium subterraneum, etc.) also produce urolithins that circulate in plasma as conjugated derivatives and are excreted in urine and feces. The production of urolithins in the rumen of cattle has been demonstrated, and isourolithin A and urolithin B were the main metabolites observed, while urolithin A seems to be mainly produced in the intestine [31]. The kinetics of the production of the different metabolites suggests that urolithin B production from isourolithin A is more favored than its production from urolithin A that seems to be an end product. The occurrence of urolithins in milk seems likely, although it has not been demonstrated so far.

In monogastric animals, urolithins are produced in rat, mouse, and pig and in all these cases the main metabolite present in feces, urine, and plasma is urolithin $\mathrm{A}$ and its glucuronide and sulfate conjugates. Urolithins $\mathrm{C}$ and $\mathrm{B}$ are less frequent, but they have also been detected together with 
TABLE 1: Production of urolithins as ellagitannin metabolites in different animals and humans.

\begin{tabular}{|c|c|c|c|}
\hline Animal production & Ellagitannin source & Metabolites & References \\
\hline Rat (Rattus norvegicus) & Pomegranate husk & Uro A, Uro B, Uro C & {$[6,20]$} \\
\hline Rat (Rattus norvegicus) & Ellagic acid & Uro A & [27] \\
\hline Rat (Rattus norvegicus) & Oak-flavored milk & Uro A, Uro B, Uro C & [28] \\
\hline Rat (Rattus norvegicus) & Pomegranate extract & Uro A & [29] \\
\hline Rat (Rattus norvegicus) & Geraniin (from Geranium thunbergii) & $\begin{array}{l}\text { Uro A, Uro M-6, Uro M-7, } \\
\text { Uro M5 }\end{array}$ & {$[30]$} \\
\hline Mouse (Mus musculus) & Pomegranate extract & Uro A & {$[24]$} \\
\hline Mouse (Mus musculus) & Pomegranate husk & Uro A & {$[21]$} \\
\hline Beaver (Castor canadensis) & Wood & Uro A; Uro B & $\begin{array}{l}{[15]} \\
{[21]}\end{array}$ \\
\hline $\begin{array}{l}\text { Complex-toothed squirrel (Trogopterus } \\
\text { xanthipes) }\end{array}$ & Unknown & Uro A & {$[16,21]$} \\
\hline Sheep (Ovis aries) & Trifolium subterraneum & Uro A, Uro B & {$[18]$} \\
\hline Sheep (Ovis aries) & Quebracho & Uro A & * \\
\hline Cattle (Bos primigenius) & Young oak leaves & Uro A, IsoUro A, Uro B & {$[31]$} \\
\hline Pig (Sus scrofa domesticus) & Acorns & Uro A, Uro C, Uro D, Uro B & [23] \\
\hline Humans (Homo sapiens) & Pomegranate juice & $\begin{array}{l}\text { Uro A, Uro C, Isouro A, } \\
\text { Uro B }\end{array}$ & $\begin{array}{l}{[7,9,25,} \\
32]\end{array}$ \\
\hline Humans (Homo sapiens) & Pomegranate extract & Uro A, UroB, Uro C & {$[10]$} \\
\hline Humans (Homo sapiens) & Walnuts & Uro A, Uro B, Uro C & $\begin{array}{c}{[8]} \\
{[25]}\end{array}$ \\
\hline Humans (Homo sapiens) & Strawberry & $\begin{array}{l}\text { Uro A, Isouro A, Uro B, } \\
\text { Uro C }\end{array}$ & {$[8,33]$} \\
\hline Humans (Homo sapiens) & Raspberry & Uro A, IsoUro A, Uro B & {$[8,34]$} \\
\hline Humans (Homo sapiens) & Blackberry & Uro A, Uro C & * \\
\hline Humans (Homo sapiens) & Cloudberry & Uro A & $*$ \\
\hline Humans (Homo sapiens) & Oak-aged red wine & Uro A & {$[8]$} \\
\hline Humans (Homo sapiens) & Tea & Uro A & [35] \\
\hline Humans (Homo sapiens) & Nuts & Uro A, Isouro A, Uro B & {$[36]$} \\
\hline
\end{tabular}

Uro A (urolithin A); Uro B (urolithin B); Uro C (urolithin C); Uro D (urolithin D); Isouro A (isourolithin A); Uro M-5 (urolithin M-5); Uro M-6 (urolithin M-6); and Uro M-7 (urolithin M-7). *Tomás-Barberán et al., unpublished results.

small amounts of isourolithin A (Table 1). The same behavior is observed in humans after the intake of pomegranates, walnuts, tea, muscadine grapes, strawberries, raspberries, blackberries, cloudberries, oak acorns, and oak-aged red wine. Urolithins will also be eventually produced after the intake of all ellagitannin-containing foods and medicinal plants as is the case of camu camu (Myrciaria dubia), arctic bramble, rose hip, sea buckthorn, cranberry, Geranium, and oak-aged spirits (whisky, etc.).

The studies to evaluate urolithin production in other animals, including birds and insects, are very limited [21], but the evidence so far indicates that they do not produce urolithins from ellagitannins. In insects, ellagitannins are hydrolyzed to release ellagic acid, which is detected in feces. This was shown in the acorn beetle (Thorectes lusitanicus) [21]. Indirect evidence shows that honeybees harvesting nectar containing ellagitannins hydrolyze them to release ellagic acid during honey maturation [37], but no urolithins are detected suggesting that honeybee microbiota is not able to metabolize ellagic acid to produce urolithins. In termites feeding on wood, ellagitannins are hydrolyzed to ellagic acid, and then hydroxyls are removed to produce nasutins, and it seems that termite microbiota does not have the ability of removing the lactone ring of ellagic acid to produce urolithins [21]. It has been reported that the hemolymph of some Australian termites of the genus Nasutitermes contains nasutin A that is also present in feces, but no urolithin was detected [38].

In birds the only study available was done with greenfinches (Carduelis chloris) that were fed for 2 weeks with blackberries. Feces were collected and analyzed, and only ellagic acid was detected [21].

These results show that urolithins are generally produced by mammals after the intake of ellagitannins. There is, however, interindividual variability that has been suggested to be associated with different gut microbiota composition, and this means that the health effects observed after the intake of pomegranates and other ellagitannin-containing food can 
TABLE 2: Biological effects of urolithins assayed on human cell lines.

\begin{tabular}{|c|c|c|c|c|c|}
\hline Test compound & Activity & Test model & Dose/duration & Effect & Ref. \\
\hline UA, UB, UC, UD & Antioxidant & $\begin{array}{l}\text { PMA } \\
\text { DCFH-DA } \\
\text { HL-60 cells }\end{array}$ & $0.04-137 \mu \mathrm{M}, 0.5 \mathrm{~h}$ & $\begin{array}{l}\text { Inhibit cellular injury caused by } \\
\text { ROS }\end{array}$ & [39] \\
\hline UA, UB & Antioxidant & $\begin{array}{l}\text { DMNQ } \\
\text { SK-N-MC cells }\end{array}$ & $0.1-20 \mu \mathrm{M}, 0.5 \mathrm{~h}$ & Increase cell survival & [40] \\
\hline UA, UB & $\begin{array}{l}\text { Estrogenic } \\
\text { Anti-estrogenic }\end{array}$ & MCF-7 cells & $0.1-40 \mu \mathrm{M}, 7 \mathrm{~d}$ & $\begin{array}{l}\text { Induction of cell proliferation } \\
\text { (estrogenic)/inhibition of cell } \\
\text { proliferation in the presence of } \\
\text { estradiol (anti-estrogenic) }\end{array}$ & {$[41]$} \\
\hline UA, UB & Antimalarial & $\begin{array}{l}\text { THP-1 stimulated with } \\
\text { haemozoin or TNF- } \alpha\end{array}$ & $25 \mu \mathrm{M}, 48 \mathrm{~h}$ & $\begin{array}{l}\text { Inhibit the release and expression } \\
\text { of MMP-9 }\end{array}$ & {$[42]$} \\
\hline $\mathrm{UA}, \mathrm{UB}$ & Anti-inflammatory & $\begin{array}{l}\text { CCD18-Co/THP-1 } \\
\text { stimulated with TNF- } \alpha\end{array}$ & $5-40 \mu \mathrm{M}, 48 \mathrm{~h}$ & $\begin{array}{l}\text { Decrease fibroblasts migration } \\
\text { and monocyte adhesion to } \\
\text { fibroblasts }\end{array}$ & [43] \\
\hline UA, UB & Anti-inflammatory & $\begin{array}{l}\text { CCD18-Co stimulated with } \\
\text { IL- } 1 \beta\end{array}$ & $0.1-40 \mu \mathrm{M}, 18 \mathrm{~h}$ & $\begin{array}{l}\text { Inhibit PGE } 2 \text { production and } \\
\text { mPGES- } 1 \text { and COX- } 2 \text { expression }\end{array}$ & {$[44]$} \\
\hline UA, UB, UAG, UBG & Anti-inflammatory & $\begin{array}{l}\text { HAOEC/THP-1 stimulated } \\
\text { with TNF- } \alpha\end{array}$ & $1-20 \mu \mathrm{M}, 4-24 \mathrm{~h}$ & $\begin{array}{l}\text { Inhibit monocyte adhesion and } \\
\text { endothelial cell migration }\end{array}$ & {$[45]$} \\
\hline UA, UB, UC, UD & Anticancer & 22Rv1 EROD assay & $6.75-50 \mu \mathrm{M}, 0.5,24 \mathrm{~h}$ & $\begin{array}{l}\text { Inhibit CYP1B1 and CYP1A1 } \\
\text { activity }\end{array}$ & {$[46]$} \\
\hline UA, UB & Anticancer & Caco-2 cells & $40 \mu \mathrm{M}, 24-96 \mathrm{~h}$ & $\begin{array}{l}\text { Cell cycle arrest in } S \text { and } \mathrm{G}_{2} / \mathrm{M} \\
\text { phases }\end{array}$ & [47] \\
\hline UA & Anticancer & $\begin{array}{l}\text { HEK T293 cells Wnt } \\
\text { luciferase assay }\end{array}$ & $\begin{array}{l}0.2-200 \mu \mathrm{g} / \mathrm{mL} \\
48 \mathrm{~h}\end{array}$ & Wnt pathway inhibition & {$[48]$} \\
\hline UA, UB, UC, UD & Anticancer & HT-29 cells & $25-70 \mu \mathrm{M}$ & $\begin{array}{l}\text { Apoptosis induction and CYP1 } \\
\text { activity inhibition }\end{array}$ & [49] \\
\hline
\end{tabular}

Caco-2: human colon carcinoma cell line; CCD18-Co: human colon fibroblast cell line; CYP1A1: cytochrome P450, family 1, member A1; CYP1B1: cytochrome P450, family 1, member B1; DCFH-DA: $2^{\prime}, 7^{\prime}$-dichlorodihydrofluorescein diacetate; DMNQ: 2,3-dimethoxy-1,4-naphthoquinone; EROD: ethoxyresorufin-Odeethylase assay; HAOEC: human aortic endothelial cell line; HEK T293: human embryonic kidney cell line; HL-60: promyelocytic leukaemia cells; HT-29 human colon carcinoma cell line; MCF-7: human breast cancer cell line; PGE2: prostaglandin E2; PMA: phorbol 12-myristate-13-acetate; SK-N-MC: human neuroblastoma cells; THP-1: human acute monocytic leukaemia cell line; UA: urolithin A; UAG: urolithin A glucuronide; UB: urolithin B; UBG: urolithin B glucuronide; UC: urolithin C; UD: urolithin D; 22Rvl: human prostate carcinoma cell line.

be modulated by the occurrence of specific microbiota to produce urolithins $[50,51]$.

\section{Biological Activity of Urolithins}

The bioavailability and metabolism studies clearly indicate which metabolites should be tested and at what concentrations in the mechanistic studies to understand the biological activity of pomegranate ellagitannins. A combination of punicalagin, ellagic acid and urolithins should be tested in those studies using models of gastrointestinal tract cells where they can reach concentrations around several hundred $\mu \mathrm{M}$ (for punicalagin and ellagic acid) and tens $\mu \mathrm{M}$ for urolithins.

The health effects attributed to urolithins based on studies carried out in vitro are numerous and diverse, from antimalarial properties or topoisomerase inhibitors to quenchers of bacterial quorum sensing. In general, the number of publications regarding each biological activity is still very limited, although these studies are generally carried out using a suitable physiological concentration range, in the order of submicromolar-low micromolar concentrations, similar to those that urolithins can reach in the gut and plasma after the ingestion of ellagitannin-rich foods (Table 2). From all the studies carried out in vitro, it is important to highlight a recent study in which the biological effects of urolithin glucuronides, the major circulating metabolites in plasma after ingestion of ellagitannin-rich foods, were explored in human aortic endothelial cells [45]. This type of study is still very scarce, although highly desirable, when studying the mechanism of action of polyphenols or their metabolites outside the gastrointestinal tract, as polyphenols and their gut microbiota metabolites are glucuronidated by Phase II enzymes after absorption, and they are found in this conjugated form in the circulatory system and peripheral tissues.

5.1. Antioxidant Activity. Given the high antioxidant properties of ellagitannin-rich foods, as is the case of pomegranate [1], one of the first activities that had been explored for 
urolithins was their antioxidant capacity. The first available study reported that urolithin A had an antioxidant capacity 42-times lower than that of its precursor punicalagin when it was tested in the DPPH assay and 3,500 times lower when it was tested in the ABTS assay [7]. These results were consistent with the concentrations of urolithins necessary to reach an $\mathrm{IC}_{50}$ in several antioxidant assays $(\mathrm{DPPH}$, xanthine-XOD, and PMS-NADH) that were above $100 \mu \mathrm{M}$ [29]. However, using the ORAC assay, all urolithins tested exhibited potent antioxidant properties compared with those of ascorbic acid, urolithin A being the most potent among them [29]. In a similar study, in which the antioxidant activity of a large number of polyphenols and their metabolites produced in vivo was compared by the ORAC method, the antioxidant activity of urolithin A was one of the most powerful, only beaten by some proanthocyanidin oligomers, catechin, epicatechin and 3,4-dihydroxyphenyl acetic acid [52]. Using a cell-based method, in which the transport through the cell membrane was considered, Bialonska et al. [39] determined that urolithin $\mathrm{C}$ showed the highest antioxidant power $\left(\mathrm{IC}_{50}=\right.$ $0.16 \mu \mathrm{M}$ ) whereas urolithin A showed an IC $_{50}$ of $13.6 \mu \mathrm{M}$, still in the range of the plasma concentrations achievable in vivo but in a lesser degree than its precursor ellagic acid $(1.1 \mu \mathrm{M})$ or vitamin $\mathrm{C}(1.9 \mu \mathrm{M})$ [39]. In neuronal cells in which an oxidative stress was induced, urolithin $\mathrm{B}(0.5-20 \mu \mathrm{M})$ and urolithin $\mathrm{A}(10 \mu \mathrm{M})$ exhibited a protective effect increasing cell survival [40]. In general, it is assumable that urolithins have higher antioxidant capacity than was originally thought but the antioxidant capacity depends on the measurement method and needs to be studied in more detail and with other in vivo methods given the importance of free radicals in many diseases.

5.2. Estrogenic Modulators. In the last few years, there has been an increasing interest in the study of the estrogenic/antiestrogenic activity of plant-derived compounds (phytoestrogens) due to their potential benefits as part of the diet, that is, regulation of cholesterol levels or maintenance of the bone density after menopause. Many polyphenols (isoflavones, flavanones, estilbenes, etc.) show phytoestrogenic effects. In some cases, dietary polyphenols may be the precursors of the so-called enterophytoestrogens that are produced by the colon microbiota by catabolism of the original phenolics. The potential activity of urolithins as enterophytoestrogens has recently been investigated [41]. In this work, structure-activity studies revealed that the urolithins A and B had specific molecular characteristics that made these molecules potentially able to bind with the $\alpha$ and $\beta$-estrogenic receptors (ER) [41]. The ER Competitive Binding Assays showed that both urolithins had an affinity for the ER $\alpha$ and $E R \beta$ receptors, that urolithin A bound more effectively than urolithin $B$, and that the affinity was higher for the $\mathrm{ER} \alpha$ than for the $\mathrm{ER} \beta$ receptor. Using a proliferation assay with cells sensitive to estrogens (E-screen) urolithins A and $\mathrm{B}$ exhibited estrogenic activity (in the absence of estradiol) and antiestrogenic activity (in the presence of estradiol) in a similar fashion to other known phytoestrogens [41].

\subsection{Anti-Inflammatory-Related Activities}

5.3.1. Antimalarial Activity. In an attempt to clarify whether the properties of the sun-dried rind of immature Punica granatum, that is, used as an anti-malarial herbal remedy, are at least in part due to urolithins, their activity inhibiting the MMP-9 enzyme (directly involved in the pathogenesis of malaria) was tested. Urolithins A and B at concentrations of $25 \mu \mathrm{M}$ inhibited the release of MMP- 9 and its mRNA expression, in hemozoin and TNF- $\alpha$ stimulated monocytic cells [42]. These results indicated that the antimalarial properties of pomegranate rind may partly be due to urolithins.

5.3.2. Histone Acetylation Status. Histone acetylation/ deacetylation status plays an important role in inflammation since it is associated with the activation/deactivation of transcription factors as NF- $\kappa$ B and AP-1, directly implicated in inflammation. One of the mechanisms by which urolithins exert their anti-inflammatory activity could be the inhibition of histone acetyltransferases (HAT) as in fact has been demonstrated for $5 \mu \mathrm{M}$ urolithin $\mathrm{A}$ and B that were able of inhibiting HAT activity [53].

\subsubsection{Anti-Inflammatory Effects on Human Colon Fibroblasts.} Colon fibroblasts have an important role in the gut immune response and can be exposed to significant quantities of colon dietary metabolites. Following ellagitannins intake, urolithin A and urolithin B as well as traces of ellagic acid can be found in the intestine. Thus, cultured human colon fibroblasts stimulated with proinflammatory cytokines were exposed to a mixture of urolithins $\mathrm{A}$ and $\mathrm{B}$ and ellagic acid at concentrations representative of those that may be found in vivo (5-40 $\mu \mathrm{M})$ [43]. Urolithin A and, most significantly, the mixture of metabolites were able to inhibit the migration capacity of the fibroblasts and the adhesion of monocytes to the fibroblasts giving evidence of a potential amelioration of inflammation in the colon cells [43]. Further molecular insights into these responses revealed that these effects were concomitant with a significant downregulation of the levels of prostaglandin-E2 (PGE2), PAI-1, and interleukin 8 (IL-8) as well as of other key regulators of cell migration and adhesion (Table 2). The study of the individual metabolites indicated that urolithin A was the most active compound. In addition to the inhibition of PGE2, urolithin A and also urolithin B were able to inhibit the expression of the two major enzymes responsible for the synthesis of prostaglandins under inflammatory conditions (mPGES-1 and COX-2) whereas ellagic acid did not show any effect [44]. The anti-inflammatory effects of urolithins may be mediated through regulation of the transcription factor NF- $\kappa \mathrm{B}$ since both urolithins are able to inhibit the activation of this factor and also exhibit an inhibitory effect on the mitogen-activated protein kinase pathways (MAPK pathways), c-Jun (urolithin A), and p38 (urolithins A and B) [44]. 
5.3.4. Anti-Inflammatory Effects on Human Endothelial Cells. The anti-inflammatory effects of urolithin glucuronides have recently been investigated by Giménez-Bastida et al. [45]. Using cytokine-induced human aortic endothelial cells, this study explored the effects of physiologically relevant concentrations (low $\mu \mathrm{M}$ range, $1-20 \mu \mathrm{M}$ ) of urolithin $\mathrm{A}$ and $\mathrm{B}$ glucuronides (the main metabolites detected in human plasma after the oral intake of ellagitannin-containing foods [7]) on two early atherosclerotic events: monocyte adhesion to endothelial cells and endothelial cell migration. The main outcome of this research was that urolithin A glucuronide, the most abundant circulating conjugate after the intake of ellagitannins, exhibited the highest anti-inflammatory activity. Urolithin A glucuronide moderately but significantly inhibited monocyte adhesion to the endothelial cells as well as the migration capacity of these endothelial aortic cells [45]. These effects were associated with a moderate but significant regulation of the levels of several key molecular markers associated with the atherosclerotic process: (i) downregulation of chemokine (C-C motif) ligand 2 (CCL2), (ii) downregulation of plasminogen activator inhibitor-1 (PAI-1), and (iii) regulation of several growth factors. These results were, in some cases, comparable to those observed for the corresponding aglycone urolithin $\mathrm{A}$ and suggested that the glucuronidation of urolithin A does not entirely eliminate the activity of the aglycone and that the glucuronide may contribute to the beneficial effects against cardiovascular diseases attributed to the consumption of pomegranate (or pomegranate juice) [22] and other ellagitannin-containing foods [2].

\subsection{Anticarcinogenic-Related Activities}

5.4.1. Topoisomerase II and CK2 Inhibitors. The anticarcinogenic activity of urolithins is one of the most explored so far. Urolithins seem to exert anticarcinogenic activity by affecting numerous molecular pathways [5]. Urolithins are inhibitors of the CK2 enzyme, a ubiquitous protein kinase implicated in a wide variety of cell functions that when altered lead to processes like inflammation and cancer. Using submicromolar concentrations in in silico screening, urolithin A was discovered to be a potent and selective CK2 inhibitor $\left(\mathrm{IC}_{50}=0.39 \mu \mathrm{M}\right)$ [54]. Urolithin M5 and another synthetic urolithin (never detected as an ellagic acid or ellagitannin metabolite in animals) showed topoisomerase inhibitory activity at concentrations below $1 \mu \mathrm{M}$, showing an even more potent activity than that of the chemotherapeutic drug doxorubicin. The molecular mechanism of action toward human topoisomerase II seems to be the competition with ATP for the ATP binding pocket of the human enzyme [55].

5.4.2. Human Prostate Cells. The human prostate gland is one of the organs where urolithins can be detected after the consumption of pomegranate juice and walnuts [25]. In prostate cancer cells, urolithins (A, B, C, and D) inhibited CYP1B1 activity (a target in prostate cancer chemoprevention) in a dose ranging from $1.15 \mu \mathrm{M}$ (urolithin A) to $137 \mu \mathrm{M}$ (urolithin D) whereas higher concentrations were needed for CYP1A1 activity inhibition $(12.4-2,907 \mu \mathrm{M})$. The changes in $V_{\max }$ and $K_{m}$ parameters with increasing concentrations of CYP1B1 inhibitor suggested an uncompetitive inhibition for urolithin A. The lack of changes for these parameters, however, suggested a noncompetitive inhibition for urolithin B. Furthermore, the decrease in CYP1B1 activity exerted by urolithins was accompanied by a decrease in CYP1B1 expression [46].

5.4.3. Anticancer Effects against Human Colon Cancer Cells. The in vitro studies conducted in colon cancer cells are of great relevance since it is in this portion of the GI tract where urolithins are produced and can reach bioactive concentrations. A mixture of urolithin A, urolithin B, and ellagic acid, at concentrations representative of those attainable in the intestine through the diet, inhibited the proliferation of the human colon cancer Caco-2 cells [47]. This inhibition was mostly mediated through an $S$ and $\mathrm{G}_{2} / \mathrm{M}$ cell cycle arrest in association with the modulation of the expression of genes involved in cell cycle regulation (CCNB1 and CCNB1IP1) and in cancer development, such as the oncogenes $K$-Ras and $c-M y c$, the tumor suppressors DUSP6 and Fos, and the growth factors receptors FGFR2 and EGFR [47]. These results are concomitant with a general regulation of the ERK1/2 signaling pathway. Another pathway that may be a target for urolithins is the Wnt pathway. Using a luciferase reporter of the canonical Wnt pathway in HEK T293 colon cells, Sharma et al. [48] showed that urolithin A was able to inhibit Wnt signaling with an $\mathrm{IC}_{50}$ of $39 \mu \mathrm{M}$ whereas ellagic acid showed an $\mathrm{IC}_{50}$ of $63 \mu \mathrm{M}$, concentrations that can be achieved in the colon after pomegranate or ellagitanninrich foods consumption [48]. Kasimsetty et al. [49] also tested and compared the effects of urolithins on human colon cancer HT-29 cells, and found that urolithins A, B, C, and $\mathrm{D}$ were able to induce apoptosis in a concentration range between 25 and $50 \mu \mathrm{M}$, whereas much higher concentrations $(\sim 500 \mu \mathrm{M})$ were necessary to promote cell cycle arrest. Urolithins A, B, C, and D also inhibited CYP1 activity (by approximately $50 \%$ ) using concentrations in the range of 50 to $75 \mu \mathrm{M}$. All these results suggest that regular dietary consumption of ellagitannin-containing foods, which can yield and maintain $\mu \mathrm{M}$ concentrations of urolithins in the colon, may have a protective effect against colon cancer development.

5.5. Protein Glycation Inhibitors. The production of advanced glycation end products is a secondary effect of hyperglycemia that has a significant role in the cardiovascular complications associated with diabetes and also with Alzheimer's disease. Urolithins A and B $(1 \mu \mathrm{M})$ showed significant antiglycative activity that increased when increasing the concentrations up to $10 \mu \mathrm{M}$ in the case of urolithin A. The same increase was not observed for urolithin B [40]. This activity, however, was not related to their antioxidant activity (measured as ABTS) or to their glyoxal-binding capacity. 
5.6. Antimicrobial Activity through the Inhibition of Quorum Sensing. Quorum Sensing (QS) is a bacterial communication mechanism that responds to small molecules or autoinducers and by which bacteria are able to detect population density, regulate gene expression, and control several key processes related to the infection progression such as virulence, biofilm formation, and motility. An important area of recent research focuses on finding natural compounds able to inhibit QS that may pose an efficient alternative to the use of antibiotics against pathogen infections. Dietaryderived polyphenol metabolites present in the intestine may contribute to reduce the ability of pathogens to invade the intestine through inhibition of their QS capacity. Urolithins $A$ and $B$ have been shown to reduce biofilm biomass and swimming motility of the enteropathogen Yersinia enterocolitica at concentrations as low as $4 \mu \mathrm{M}$ [56]. These effects were associated with a significant reduction of the levels of the bacteria autoinducers acylhomoserine lactones (AHLs) released by the bacteria to the culture media and were accompanied by the alteration of the expression levels of genes critically involved in the synthesis of lactones (yenI and $y e n R$ ) and the synthesis of the flagella ( $f h D C$, fliA, and $f l e B)$ [56]. These results suggest that the microbiotaderived metabolites urolithin A and urolithin B may exert antipathogenic effects in the colon against $Y$. enterocolitica and may contribute to maintain the microbial equilibrium in the gut.

\section{The In Vivo Evidence}

The poor bioavailability of ellagitannins, and ellagic acid derivatives as well as their extensive metabolism in the gastrointestinal tract has raised the question whether these parent molecules, found as such in the food, were the real active compounds systemically that could be related to the health benefits exerted by pomegranate juice and other ellagitannin-containing foodstuffs. Our group reported for the first time the occurrence of urolithins in humans after consuming pomegranate juice [7]. These metabolites reached micromolar concentrations in the bloodstream, and we launched the hypothesis that linked the potential systemic biological effects of pomegranate juice ingestion with urolithins rather than with the polyphenols present in pomegranate juice [7] or other ellagitannin-containing foodstuffs such as walnuts, strawberries, and raspberries [8].

The first direct in vivo evidence regarding the biological activity of urolithins was also reported by our group five years later after launching the above hypothesis. Larrosa et al. [29] reported the anti-inflammatory and prebiotic effects of the most significant urolithin metabolite (urolithin A) in a rat model of ulcerative colitis. The rats received a standard chow supplemented either with $250 \mathrm{mg} / \mathrm{kg}$ /day of an ellagitannin-rich pomegranate extract (PE) (HED $\sim 2.5 \mathrm{~g}$ in a $70 \mathrm{~kg}$ person) or with $15 \mathrm{mg} / \mathrm{kg} /$ day of synthetic urolithin A (UroA) (HED $\sim 154 \mathrm{mg}$ in a $70 \mathrm{~kg}$ person) for 25 days before inducing colon inflammation with dextran sodium sulfate (DSS). The pomegranate extract contained $35 \%$ punicalagins, $13 \%$ punicalin, $4.5 \%$ ellagic acid glycosides, and $8.9 \%$ free ellagic acid. Taking into account that urolithin $\mathrm{A}$ is the main metabolite produced after pomegranate intake, the objective of that study was to evaluate a possible direct effect of urolithin A ingested as synthetic compound and to compare this effect with that exerted by the in vivo generated urolithin A after pomegranate extract consumption. A number of measurements were carried out, including the evaluation of colon tissue damage, microbiota changes, antioxidant status, prostaglandin E2 (PGE2), nitric oxide production, cyclooxygenase-2 (COX-2), inducible nitric oxide synthase (iNOS), prostaglandin E synthase (PTGES), gene expression in colon mucosa (microarrays and RT-PCR) and polyphenol metabolism (LC-MS-MS). The administration of both pomegranate extract and urolithin A for 25 days before the induction of inflammation was reported to be safe according to serobiochemical analysis and evaluation of the animals. Both pomegranate extract and urolithin-A decreased the anti-inflammatory markers iNOS, COX-2, PTGES and PGE2 in colonic mucosa. However, the anti-inflammatory activity exerted by $15 \mathrm{mg} / \mathrm{kg}$ urolithin A at both colonic and systemic levels was relatively stronger than that produced by $250 \mathrm{mg} / \mathrm{kg}$ pomegranate extract. Only urolithin A was able to preserve the colonic architecture. However, pomegranate extract but not urolithin A decreased oxidative stress in plasma and colon mucosa (TBARs and FRAP methods).

Regarding gene expression analyses in the colon mucosa of a rat model of ulcerative colitis, the expression of 2,058 and 6,996 genes was found to be significantly modified by the consumption of the pomegranate extract and urolithin $\mathrm{A}$, respectively. From those, a total of 667 genes were commonly regulated both by the pomegranate extract and urolithin A. These genes were used for Functional Analyses using the Ingenuity Pathways Analysis (IPA, Ingenuity Systems, Redwood City, CA, USA). Figure 5 shows the main top functions and diseases that were most significant to the gene expression altered by the intake of the pomegranate extract or urolithin A. Of note, some of the main functions and disorders detected were associated to colon cancer development, for example, "cell death," "cell proliferation," "cancer," "gastrointestinal disease," "organismal survival," and "cell cycle."

Using the Canonical Pathways Analysis tool of IPA, it was also possible to identify the main pathways from the IPA library that were most significant to the gene expression data analyzed. Figure 6 shows the main molecules specifically involved in signaling pathways, transcription regulation, cell growth, cell cycle, and apoptosis for which deregulation has been implicated in cancer development and whose transcript levels were significantlymodulated by urolithin A (upregulated in red, downregulated in green). The different molecules are depicted in their corresponding cellular compartment, and reported interactions between them are indicated by arrows. This figure illustrates the complexity of the molecular mechanisms that may be triggered in a cell as a response to the exposure to a potential bioactive compound, for example, urolithin A. It should be noted that among the changes detected, some important tumor suppressors such as p53 and $\mathrm{Rb} 1$ were upregulated whereas the antiapoptotic genes $\mathrm{Bcl}_{\mathrm{XL}}$ 


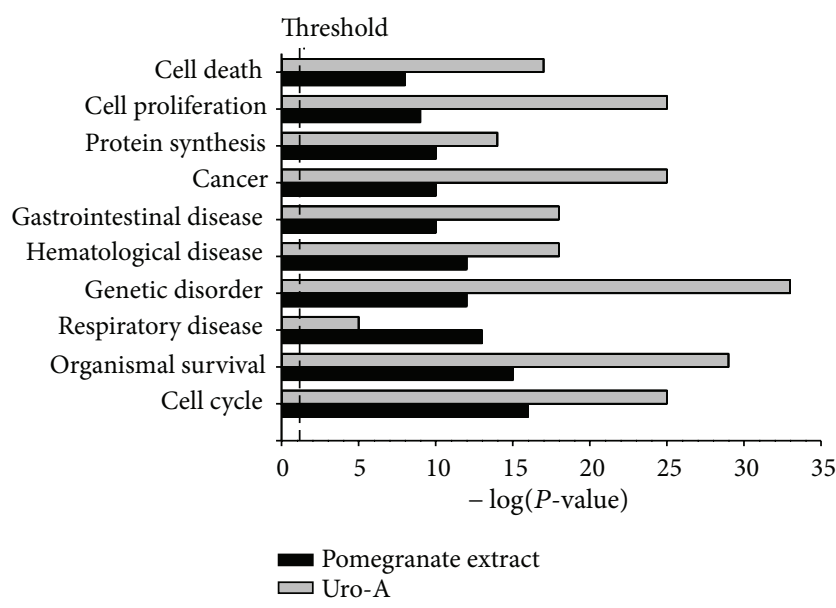

Figure 5: Common top ten functions and diseases identified by functional analysis (IPA, Ingenuity Pathways Analysis; Ingenuity Systems, Redwood City, CA, USA) and that were most significant to gene expression altered by the intake of pomegranate extract or Urolithin A in the colon mucosa of a rat model of ulcerative colitis (gene expression data from Larrosa et al. [29]). Black bars: pomegranate extract. Grey bars: urolithin A. A Fischer's exact test was used to calculate a $P$-value determining the probability that each function or disease assigned to the data was due to chance. We set a threshold at $P$ value $\leq 0.05$ (dashed line) which corresponds to a False Discovery Rate (FDR) $\leq 5 \%$ of false positives. Note: On the $y$ axis, the significance is expressed as the minus log (10-based) of the $P$ values. The higher the bar, the lower the $P$ value and hence the more significant for the enrichment of the function/disease.

and Akt were downregulated by urolithin A. These changes were also observed after treatment with the pomegranate extract. These results are indicative of in vivo molecular modulation in the colon mucosa cells in response to urolithin A or pomegranate extract that may be associated with the prevention or reduction of malignant changes and cancer development. In support of these results, in vitro studies using human colon cancer cells Caco-2 also reported the inhibition of these cells proliferation and a cell cycle block by urolithin A (Table 2). In addition, many of those genes involved in these functions were found to be modulated by urolithin $\mathrm{A}$ in the human cells [47].

Our group also reported for the first time the in vivo prebiotic effect of pomegranate extract and urolithin A [29]. Both pomegranate extract and urolithin A modulated favorably the gut microbiota in healthy rats (before inflammation) which could contribute to the protective effects of pomegranate juice and urolithin A against the further induced colon inflammation. In the case of urolithin $\mathrm{A}$, the effect was unequivocally due to synthetic metabolite orally administered and could be associated to the inhibition of some specific gut microbiota species to favor the growing of some (beneficial) microbial groups such as lactobacilli and bifidobacteria. In the case of pomegranate extract, the same explanation could be also given, but a prebiotic effect exerted by other compounds present in the extract (fibers, sugars, etc.) could not be ruled out [29].
The second study dealing with the in vivo evidence of urolithins was carried out by Ishimoto et al. [57]. These authors reported the acute $(24 \mathrm{~h})$ anti-inflammatory effect of urolithin A on carrageenan-induced paw edema in mice. In this case, urolithin A $(300 \mathrm{mg} / \mathrm{kg}$; HED $\sim 1.5 \mathrm{~g}$ in a $70 \mathrm{~kg}$ person) was orally administered to mice at 1 or $6 \mathrm{~h}$ before the injection of carrageenan. The inflammatory effect was evaluated for $24 \mathrm{~h}$ after carrageenan injection by measuring the hind paw volume. Unfortunately no inflammatory markers were measured in this study. The authors claimed a rapid absorption of urolithin A which peaked at $1 \mathrm{~h}$ in mice [57]. However, this was not in agreement with a previous study dealing with the pharmacokinetic and tissue distribution of urolithins where urolithin A peaked in plasma after $2 \mathrm{~h}$, being almost undetectable after $6 \mathrm{~h}$ [24]. Ishimoto et al. [57] described a high antioxidant activity of urolithin A in mouse plasma using the ORAC method and suggested that this activity could be correlated with the anti-inflammatory effects. These results were not in agreement with those of Larrosa et al. [29] who suggested that anti-inflammatory effects of urolithin A were not mediated by its antioxidant activity since no significant antioxidant effects in plasma and colon of rats were found (TBARs and FRAP methods). In addition, Cerdá et al. [7] did not observe any increase in the antioxidant status of human plasma after the intake of $1 \mathrm{~L}$ of pomegranate juice for 5 days (ABTS and DPPH methods). Perhaps, the high dose of urolithin A assayed by Ishimoto et al. [57] as well as the specific use of the ORAC method to measure the antioxidant activity could be behind this apparent controversy. Overall, the human extrapolation of the results obtained by Ishimoto et al. [57] could be doubtful due to their assay conditions. These authors assayed a single dose of $1.5 \mathrm{~g}$ HED of urolithin A which raises safety concerns in contrast to the assay conditions approached by Larrosa et al. [29].

\section{Outlook and Further Research}

Urolithins can reach significant concentrations in the human body after the intake of ellagitannin-containing products; however, the direct biological activity of urolithins has been scarcely studied so far. In this regard, these metabolites could be the missing link to explain the health benefits associated to the consumption of ellagitannin-containing products such as pomegranate. There are a number of urolithins produced by the human gut microbiota. Some studies reveal the relevant turnover of these metabolites in the human body, with a high urine excretion, significant concentration in the bloodstream, and also disposition in the human prostate gland. To date, only two studies (animal models) have described the in vivo anti-inflammatory $[29,57]$ and possible cancer chemopreventive activities [29] of the most abundant urolithin produced in humans, urolithin $\mathrm{A}$. In addition to this limited evidence, the direct in vivo activity of the rest of urolithins is not yet known, and thus more animal studies are needed. In vitro studies with specific cell models and specific metabolites (type of urolithin and conjugates such as glucuronides or sulfates) are useful to unravel mechanisms. In this context, 
Molecular mechanisms of cancer

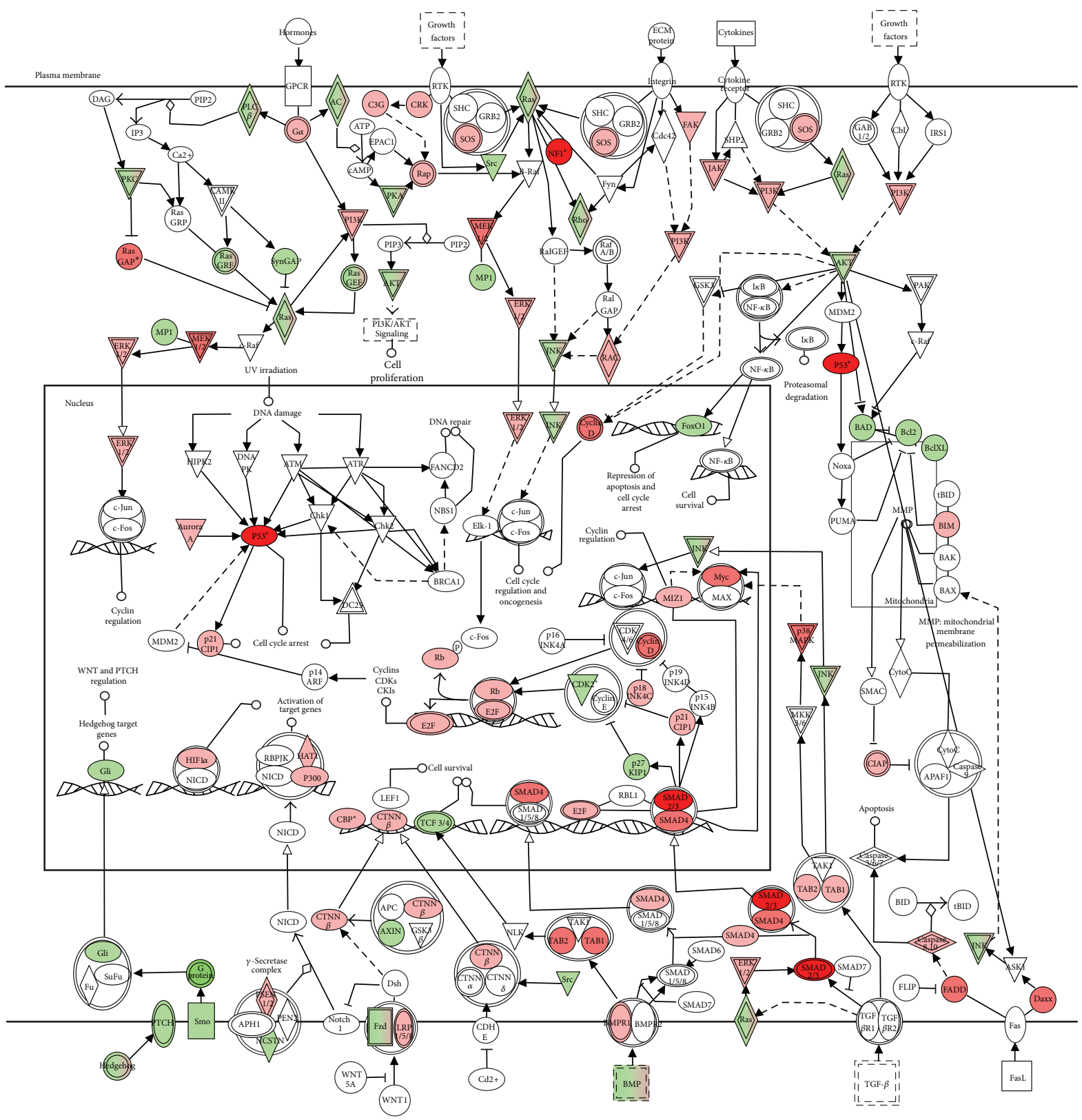

(c) 2000-2010 Ingenuity systems, Inc. all rights reserved.

FIGURE 6: Graphical representation of main genes involved in signaling pathways, transcription regulation, cell growth, cell cycle, and apoptosis for which deregulation has been implicated in cancer development. Genes depicted in red color were upregulated and genes represented in green color were downregulated in the colon mucosa of rats following the consumption of urolithin A, (Larrosa et al. [29]). Analyses were carried out using the Canonical Pathways Analysis tool of Ingenuity Pathways Analysis (Ingenuity Systems, Redwood City, CA, USA).

appropriate concentrations and type of metabolites should be carefully chosen to draw relevant conclusions from these in vitro models.

The evaluation of urolithins in humans could be more difficult. Although these metabolites are produced in the human gut, the administration of synthetic urolithins to humans can raise safety concerns since these metabolites are not found as such (at least in relevant concentrations) in foodstuffs, and therefore they cannot be considered as dietary compounds. In this regard, the toxicological evaluation of urolithins in animal models is also missing.

\section{Conflict of Interests}

The authors declare no conflict of interests. 


\section{Acknowledgments}

This work has been supported by the Projects Consolider Ingenio 2010, CSD2007-00063 (Fun-C-Food), CICYT AGL2011-22447, Fundación Séneca de la Región de Murcia (grupo de excelencia GERM 06, 04486), and BACCHUS (FP7 European Commission Grant Agreement 312090).

\section{References}

[1] M. I. Gil, F. A. Tomás-Barberán, B. Hess-Pierce, D. M. Holcroft, and A. A. Kader, "Antioxidant activity of pomegranate juice and its relationship with phenolic composition and processing," Journal of Agricultural and Food Chemistry, vol. 48, no. 10, pp. 4581-4589, 2000.

[2] M. Larrosa, M. T. García-Conesa, J. C. Espín, and F. A. TomásBarberán, "Ellagitannins, ellagic acid and vascular health," Molecular Aspects of Medicine, vol. 31, no. 6, pp. 513-539, 2010.

[3] F. A. Tomás-Barberán, J. C. Espín, and M. T. García-Conesa, "Bioavailability and metabolism of ellagic acid and ellagitannins," in Chemistry and Biology of Ellagitannins, S. Quideau, Ed., chapter 7, pp. 293-297, World Scientific, New Yersey, NJ, USA, 2009.

[4] N. P. Seeram, R. N. Schulman, and D. Heber, Pomegranates. Ancient Roots to Modern Medicine, CRC Press, Boca Raton, FL, USA, 2006.

[5] D. Heber, "Multitargeted therapy of cancer by ellagitannins," Cancer Letters, vol. 269, no. 2, pp. 262-268, 2008.

[6] B. Cerdá, R. Llorach, J. J. Cerón, J. C. Espín, and F. A. TomásBarberán, "Evaluation of the bioavailability and metabolism in the rat of punicalagin, and antioxidant polyphenol of pomegranate juice," European Journal of Nutrition, vol. 42, no. 1, pp. 18-28, 2003.

[7] B. Cerdá, J. C. Espín, S. Parra, P. Martínez, and F. A. TomásBarberán, "The potent in vitro antioxidant ellagitannins from pomegranate juice are metabolised into bioavailable but poor antioxidant hydroxy-6H-dibenzopyran-6-one derivatives by the colonic microflora of healthy humans," European Journal of Nutrition, vol. 43, no. 4, pp. 205-220, 2004.

[8] B. Cerdá, F. A. Tomás-Barberán, and J. C. Espín, “Metabolism of antioxidant and chemopreventive ellagitannins from strawberries, raspberries, walnuts, and oak-aged wine in humans: identification of biomarkers and individual variability," Journal of Agricultural and Food Chemistry, vol. 53, no. 2, pp. 227-235, 2005.

[9] N. P. Seeram, S. M. Henning, Y. Zhang, M. Suchard, Z. Li, and D. Heber, "Pomegranate juice ellagitannin metabolites are present in human plasma and some persist in urine for up to 48 hours," Journal of Nutrition, vol. 136, no. 10, pp. 2481-2485, 2006.

[10] S. U. Mertens-Talcott, P. Jilma-Stohlawetz, J. Rios, L. Hingorani, and H. Derendorf, "Absorption, metabolism, and antioxidant effects of pomegranate (Punica granatum L.) polyphenols after ingestion of a standardized extract in healthy human volunteers," Journal of Agricultural and Food Chemistry, vol. 54, no. 23, pp. 8956-8961, 2006.

[11] F. A. Tomás-Barberán, N. P. Seeram, and J. C. Espín, "Bioavailability of pomegranate polyphenols," in Pomegranates. Ancient Roots to Modern Medicine, N. P. Seeram, R. N. Schulman, and D. Heber, Eds., Chapter 3, pp. 45-60, CRC Press, Boca Raton, FL, USA, 2006.

[12] B. Cerdá, P. Periago, J. C. Espín, and F. A. Tomás-Barberán, "Identification of urolithin $\mathrm{A}$ as a metabolite produced by human colon microflora from ellagic acid and related compounds," Journal of Agricultural and Food Chemistry, vol. 53, no. 14, pp. 5571-5576, 2005.

[13] M. A. M. Nawwar and A. M. A. Souleman, "3,4,8,9,10Pentahydroxy-dibenzo[b,d]pyran-6-one from Tamarix nilotica," Phytochemistry, vol. 23, no. 12, pp. 2966-2967, 1984.

[14] M. A. M. Nawwar, S. A. M. Hussein, and I. Merfort, "NMR spectral analysis of polyphenols from Punica granatum," Phytochemistry, vol. 36, no. 3, pp. 793-798, 1994.

[15] E. Lederer, "Chemistry and biochemistry of some mammalian secretions and excretions," Journal of the Chemical Society, pp. 2115-2125, 1949.

[16] S. J. Jeong, N. Y. Kim, D. H. Kim et al., "Hyaluronidase inhibitory active $6 \mathrm{H}$-dibenzo[b,d]pyran-6-ones from the feces of Trogopterus xanthipes," Planta Medica, vol. 66, no. 1, pp. 7677, 2000.

[17] S. Ghosal, "Chemistry of shilajit, an immunomodulatory Ayurvedic rasayan," Pure \& Applied Chemistry, vol. 62, no. 7, pp. $1285-1288,1990$.

[18] G. S. Pope, "Isolation of two benzocoumarins from "clover stone" a type of renal calculus found in sheep," Biochemical Journal, vol. 93, no. 3, pp. 474-477, 1964.

[19] L. J. Filippich, J. Zhu, P. Oelrichs et al., "Hepatotoxic and nephrotoxic principles in Terminalia oblongata," Research in Veterinary Science, vol. 50, no. 2, pp. 170-177, 1991.

[20] B. Cerdá, J. J. Cerón, F. A. Tomás-Barberán, and J. C. Espín, "Repeated oral administration of high doses of the pomegranate ellagitannin punicalagin to rats for 37 days is not toxic," Journal of Agricultural and Food Chemistry, vol. 51, no. 11, pp. 3493-3501, 2003.

[21] R. González-Barrio, P. Truchado, H. Ito, J. C. Espín, and F. A. Tomás-Barberán, "UV and MS identification of urolithins and nasutins, the bioavailable metabolites of ellagitannins and ellagic acid in different mammals," Journal of Agricultural and Food Chemistry, vol. 59, no. 4, pp. 1152-1162, 2011.

[22] M. Aviram, L. Dornfeld, M. Rosenblat et al., "Pomegranate juice consumption reduces oxidative stress, atherogenic modifications to LDL, and platelet aggregation: studies in humans and in atherosclerotic apolipoprotein E-deficient mice," American Journal of Clinical Nutrition, vol. 71, no. 5, pp. 1062-1076, 2000.

[23] J. C. Espín, R. González-Barrio, B. Cerdá, C. López-Bote, A. I. Rey, and F. A. Tomás-Barberán, "Iberian pig as a model to clarify obscure points in the bioavailability and metabolism of ellagitannins in humans," Journal of Agricultural and Food Chemistry, vol. 55, no. 25, pp. 10476-10485, 2007.

[24] N. P. Seeram, W. J. Aronson, Y. Zhang et al., "Pomegranate ellagitannin-derived metabolites inhibit prostate cancer growth and localize to the mouse prostate gland," Journal of Agricultural and Food Chemistry, vol. 55, no. 19, pp. 7732-7737, 2007.

[25] A. González-Sarrías, J. A. Giménez-Bastida, M. T. GarcíaConesa et al., "Occurrence of urolithins, gut microbiota ellagic acid metabolites and proliferation markers expression response in the human prostate gland upon consumption of walnuts and pomegranate juice," Molecular Nutrition and Food Research, vol. 54, no. 3, pp. 311-322, 2010.

[26] N. P. Seeram, R. Lee, and D. Heber, "Bioavailability of ellagic acid in human plasma after consumption of ellagitannins from pomegranate (Punica granatum L.) juice," Clinica Chimica Acta, vol. 348, no. 1-2, pp. 63-68, 2004.

[27] B. Doyle and L. A. Griffiths, "The metabolism of ellagic acid in the rat," Xenobiotica, vol. 10, no. 4, pp. 247-256, 1980. 
[28] M. Azorín-Ortuño, C. Urbán, J. J. Cerón et al., "Safety evaluation of an oak-flavored milk powder containing ellagitannins upon oral administration in the rat," Journal of Agricultural and Food Chemistry, vol. 56, no. 8, pp. 2857-2865, 2009.

[29] M. Larrosa, A. González-Sarrías, M. J. Yáñez-Gascón et al., "Antiinflammatory properties of a pomegranate extract and its metabolite urolithin A in a colitis rat model and the effect of colon inflammation on the phenolic metabolism," Journal of Nutritional Biochemistry, vol. 21, no. 8, pp. 717-725, 2010.

[30] H. Ito, "Metabolites of the ellagitannin geraniin and their antioxidant activities," Planta Medica, vol. 77, no. 11, pp. 11101115, 2011.

[31] R. González-Barrio, P. Truchado, R. García-Villalba et al., "Metabolism of oak leaf ellagitannins and urolithin production in beef cattle," Journal of Agricultural and Food Chemistry, vol. 60, no. 12, pp. 3068-3077, 2012.

[32] B. Cerdá, C. Soto, M. D. Albaladejo et al., "Pomegranate juice supplementation in chronic obstructive pulmonary disease: a 5-week randomized, double-blind, placebo-controlled trial," European Journal of Clinical Nutrition, vol. 60, no. 2, pp. 245253, 2006.

[33] P. Truchado, M. Larrosa, M. T. García-Conesa et al., "Strawberry processing does not affect the production and urinary excretion of urolithins, ellagic acid metabolites, in humans. A cross-over study," Journal of Agricultural and Food Chemistry, vol. 60, no. 23, pp. 5749-5754, 2012.

[34] R. González-Barrio, C. A. Edwards, and A. Crozier, "Colonic Catabolism of ellagitannins, ellagic acid, and raspberry anthocyanins: in vivo and in vitro studies," Drug Metabolism and Disposition, vol. 39, no. 9, pp. 1680-1688, 2011.

[35] J. J. J. van der Hooft, R. C. H. de vos, V. Mihaleva et al., "Structural elucidation and quantification of phenolic conjugates present in human urine after tea intake," Analytical Chemistry, vol. 84, no. 16, pp. 7263-7271, 2012.

[36] S. Tulipani, M. Urpi-Sarda, R. García-Villalba et al., "Urolithins are the main urinary microbial-derived phenolic metabolites discriminating a moderate consumption of nuts in free-living subjects with diagnosed metabolic syndrome," Journal of Agricultural and Food Chemistry, vol. 60, no. 36, pp. 8930-8940, 2012.

[37] F. Ferreres, P. Andrade, M. I. Gil, and F. A. Tomás-Barberán, "Floral nectar phenolics as biochemical markers for the botanical origin of heather honey," Zeitschrift fur LebensmittelUntersuchung und-Forschung, vol. 202, no. 1, pp. 40-44, 1996.

[38] B. P. Moore, "Coumarin-like substances from Australian termites," Nature, vol. 195, no. 4846, pp. 1101-1102, 1962.

[39] D. Bioalonska, S. G. Kasimsetty, S. I. Khan, and D. Ferreira, "Urolithins, intestinal microbial metabolites of pomegranate ellagitannins, exhibit potent antioxidant activity in a cell-based assay," Journal of Agricultural and Food Chemistry, vol. 57, no. 21, pp. 10181-10186, 2009.

[40] E. Verzelloni, C. Pellacani, D. Tagliazucchi et al., "Antiglycative and neuroprotective activity of colon-derived polyphenol catabolites," Molecular Nutrition and Food Research, vol. 55, supplement 1, pp. S35-S43, 2011.

[41] M. Larrosa, A. González-Sarrías, M. T. García-Conesa, F. A. Tomás-Barberán, and J. C. Espín, "Urolithins, ellagic acidderived metabolites produced by human colonic microflora, exhibit estrogenic and antiestrogenic activities," Journal of Agricultural and Food Chemistry, vol. 54, no. 5, pp. 1611-1620, 2006.
[42] M. Dell'Agli, G. V. Galli, M. Bulgari et al., "Ellagitannins of the fruit rind of pomegranate (Punica granatum) antagonize in vitro the host inflammatory response mechanisms involved in the onset of malaria," Malaria Journal, vol. 9, no. 1, article 208, 2010.

[43] J. A. Giménez-Bastida, M. Larrosa, A. González-Sarrías, F. A. Tomás-Barberán, J. C. Espín, and M. T. García-Conesa, "Intestinal ellagitannin metabolites urolithins and ellagic acid ameliorate cytokine induced inflammation and associated molecular markers in human colon fibroblasts," Journal of Agricultural and Food Chemistry, vol. 60, no. 36, pp. 8866-8876, 2012.

[44] A. González-Sarrías, M. Larrosa, F. A. Toms-Barberán, P. Dolara, and J. C. Espín, "NF- $\kappa$ B-dependent anti-inflammatory activity of urolithins, gut microbiota ellagic acid-derived metabolites, in human colonic fibroblasts," British Journal of Nutrition, vol. 104, no. 4, pp. 503-512, 2010.

[45] J. A. Giménez-Bastida, A. González-Sarrías, M. Larrosa et al., "Ellagitannin metabolites, urolithin A glucuronide and its aglycone urolithin A, ameliorate TNF- $\alpha$ induced inflammation and associated molecular markers in human aortic endothelial cells," Molecular Nutrition \& Food Research, vol. 56, no. 5, pp. 784-796, 2012.

[46] S. G. Kasimsetty, D. Bialonska, M. K. Reddy, C. Thornton, K. L. Willett, and D. Ferreira, "Effects of pomegranate chemical constituents/intestinal microbial metabolites on CYP1B1 in 22Rv1 prostate cancer cells," Journal of Agricultural and Food Chemistry, vol. 57, no. 22, pp. 10636-10644, 2009.

[47] A. González-Sarrías, J. C. Espín, F. A. Tomás-Barberán, and M. T. García-Conesa, "Gene expression, cell cycle arrest and MAPK signalling regulation in Caco-2 cells exposed to ellagic acid and its metabolites, urolithins," Molecular Nutrition \& Food Research, vol. 53, no. 6, pp. 686-698, 2009.

[48] M. Sharma, L. Li, J. Celver, C. Killian, A. Kovoor, and N. P. Seeram, "Effects of fruit ellagitannin extracts, ellagic acid, and their colonic metabolite, urolithin A, on Wnt signaling," Journal of Agricultural and Food Chemistry, vol. 58, no. 7, pp. 3965-3969, 2010.

[49] S. G. Kasimsetty, D. Bialonska, M. K. Reddy, G. Ma, S. I. Khan, and D. Ferreira, "Colon cancer chemopreventive activities of pomegranate ellagitannins and Urolithins," Journal of Agricultural and Food Chemistry, vol. 58, no. 4, pp. 2180-2187, 2010.

[50] M. V. Selma, J. C. Espín, and F. A. Tomás-Barberán, "Interaction between phenolics and gut microbiota: role in human health," Journal of Agricultural and Food Chemistry, vol. 57, no. 15, pp. 6485-6501, 2009.

[51] F. A. Tomas-Barberan and C. Andres-Lacueva, "Polyphenols and health: current state and progress," Journal of Agricultural and Food Chemistry, vol. 60, no. 36, pp. 8773-8775, 2012.

[52] H. Ishimoto, A. Tai, M. Yoshimura et al., "Antioxidative properties of functional polyphenols and their metabolites assessed by an ORAC assay," Bioscience, Biotechnology and Biochemistry, vol. 76, no. 2, pp. 395-399, 2012.

[53] A. K. Kiss, S. Granica, M. Stolarczyk, and M. F. Melzig, "Epigenetic modulation of mechanisms involved in inflammation: influence of selected polyphenolic substances on histone acetylation state," Food Chemistry, vol. 131, no. 3, pp. 1015-1020, 2012.

[54] G. Cozza, A. Gianoncelli, P. Bonvini et al., "Urolithin as a converging scaffold linking ellagic acid and coumarin analogues: design of potent protein kinase CK2 inhibitors," ChemMedChem, vol. 6, no. 12, pp. 2273-2286, 2011. 
[55] V. Furlanetto, G. Zagotto, R. Pasquale, S. Moro, and B. Gatto, "Ellagic acid and polyhydroxylated urolithins are potent catalytic inhibitors of human topoisomerase II: an in vitro study," Journal of Agricultural and Food Chemistry, vol. 60, no. 36, pp. 9162-9170, 2012.

[56] J. A. Giménez-Bastida, P. Truchado, M. Larrosa et al., "Urolithins, ellagitannin metabolites produced by colon microbiota, inhibit quorum sensing in Yersinia enterocolitica: phenotypic response and associated molecular changes," Food Chemistry, vol. 132, no. 36, pp. 1465-1474, 2012.

[57] H. Ishimoto, M. Shibata, Y. Myojin et al., "In vivo antiinflammatory and antioxidant properties of ellagitannin metabolite urolithin A," Bioorganic \& Medicinal Chemistry Letters, vol. 21, no. 19, pp. 5901-5904, 2011. 


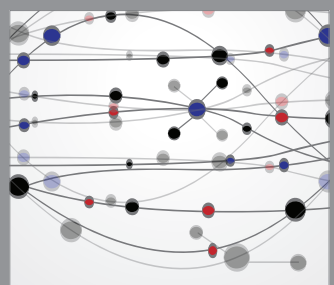

The Scientific World Journal
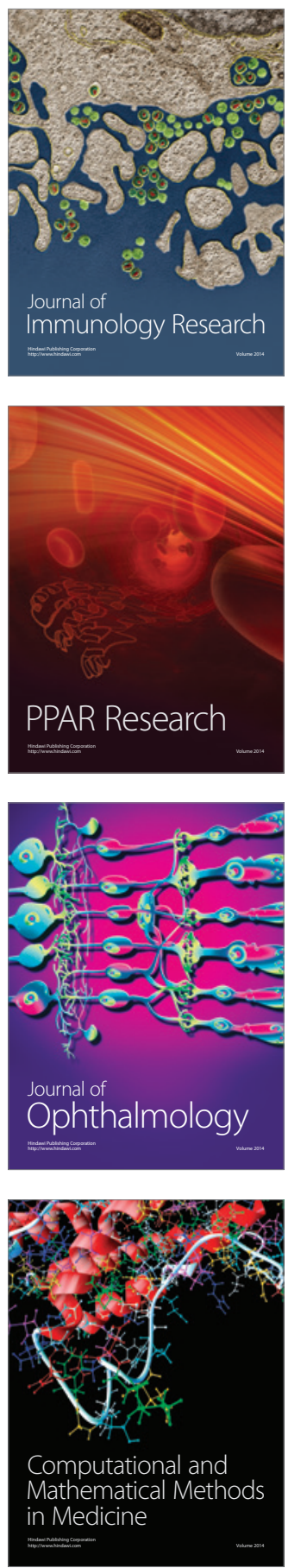

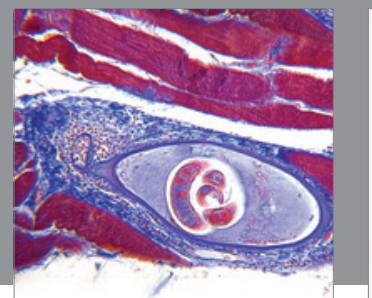

Gastroenterology

Research and Practice
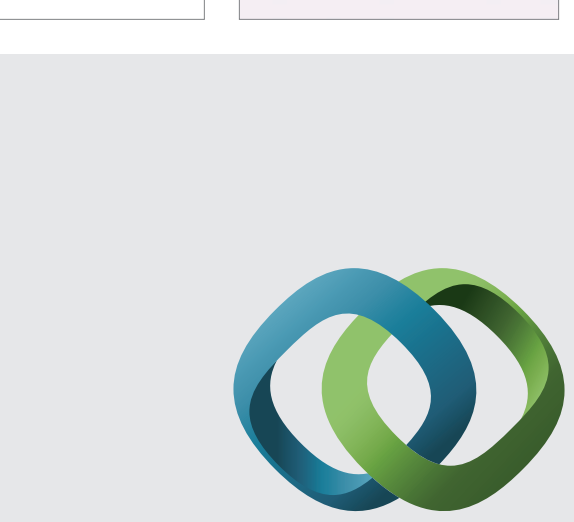

\section{Hindawi}

Submit your manuscripts at

http://www.hindawi.com
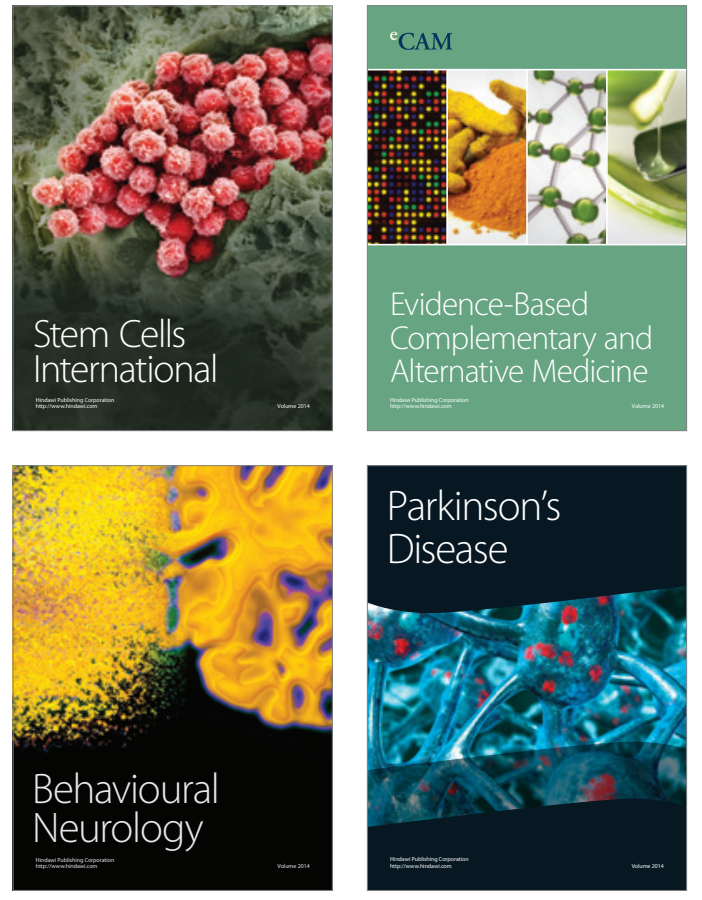
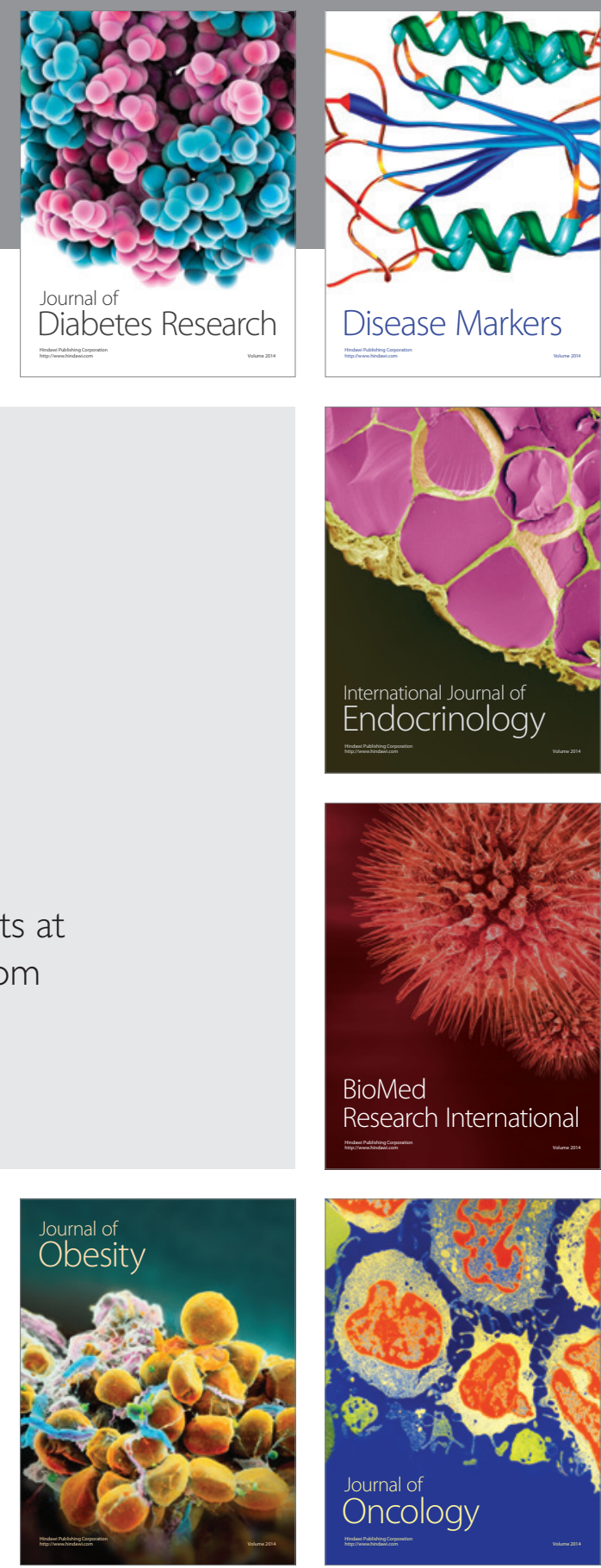

Disease Markers
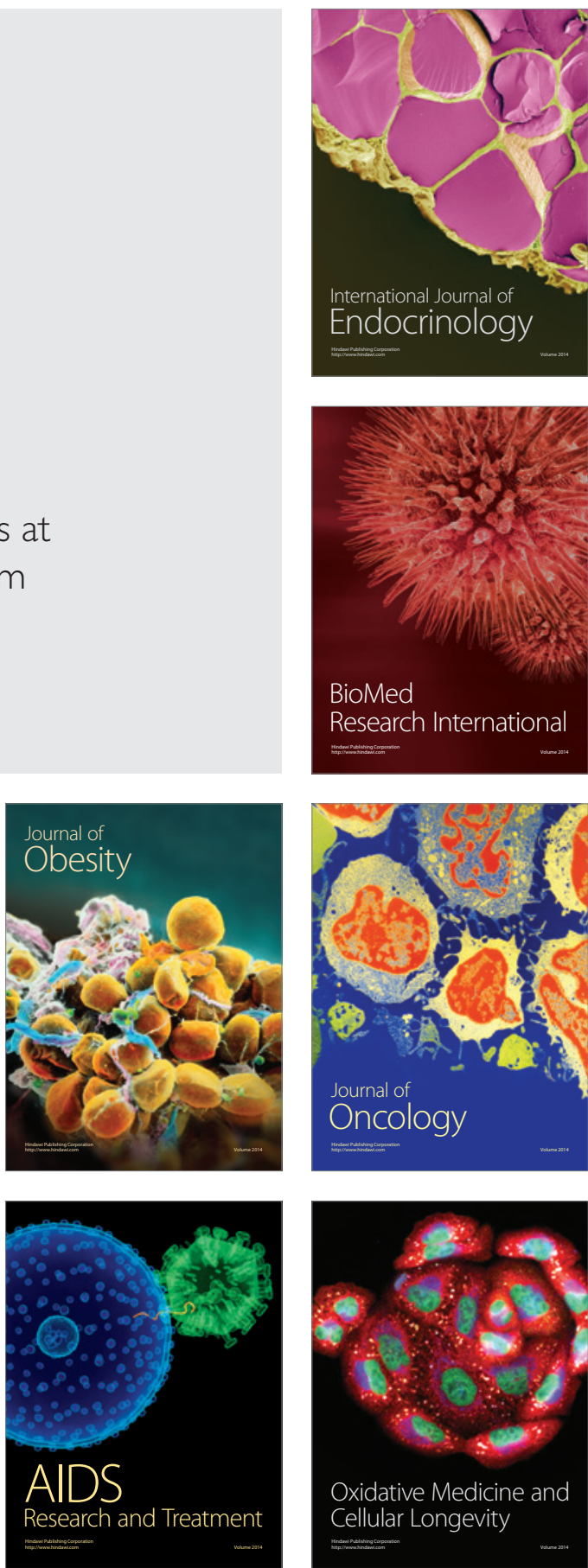